\title{
A rare case of urinary retention in an adult due to a primary penile Tumor
}

\author{
Marcelo Di Gregorio ${ }^{1 *}$, Marie Cécile Nollevaux ${ }^{2}$, Michaël Dupont $^{3}$, Pr Francis Lorge ${ }^{1}$ and Pr.Lionel D’Hondt $^{4}$ \\ ${ }^{1}$ Department of Urology, CHU UCL Namur site-Godinne, Belgium \\ ${ }^{2}$ Department of Anatomopathological, CHU UCL Namur site-Godinne, Belgium \\ ${ }^{3}$ Department of Radiology, CHU UCL Namur site-Godinne, Belgium \\ ${ }^{4}$ Department of Oncology, CHU UCL Namur site-Godinne, Belgium
}

\begin{abstract}
Background: Penile cancer is relatively rare as a primary disease in developed countries (0.81 to 1 per 100,000 persons). Penis epidermoid carcinoma is the most frequent histological presentation (93\%). Penile adenocarcinomas are most commonly metastatic disease originating from primary lesions of pelvic origin (genitourinary or recto-sigmoid tumors) but primary tumor is exceptional.
\end{abstract}

Case presentation: Here we describe a case of primary penile adenocarcinoma, which eventually metastasized to the brain. CONCLUSIONS: The rarity of the event prompted us to also explore related reviews and to discuss the incidence, physiopathology, diagnosis, and therapy of penile adenocarcinoma cancer.

\begin{abstract}
Abbreviations: NSGCTs: testicular seminomas and non-seminomatous germ cell tumours; CT: computed tomography; LDH: Lactate dehydrogenase beta-HCG Gonadotropic Chorionic Hormone; MRI: magnetic resonance imaging; ${ }^{18}$ FDG-PET: 18-fluorodeoxyglucose positron emission tomography; PLAP: Phosphatase alkaline; MMO: multidisciplinary meeting of oncology; BEP: bleomycin, etoposide, and platinum; Gy: gray
\end{abstract}

\section{Introduction}

Cancer is the most dangerous disease to haunt the mankind in the world today. Various factors are responsible for increasing cancer incidences in which modernization of our society is one of the major contributing factors [1]. The mortality rate due to cancer stands at the second positions in the world after the cardiovascular disease [2].

The reasons for most of these deaths are inefficacy and failure of the current's methods of treatments or the unviability of treatment option $[3,4]$.

Cancer of the penis is infrequent, with an annual incidence ranging from 0.81 to 1 per 100,000 persons in developed countries [5]. Penile tumors account for only $0.5 \%$ of all malignant tumors in males and are the rarest tumors of the genitourinary system. Epidermoid carcinoma is the most frequent histologic presentation (93\%) [6]. Other less common histological presentations include basal cell carcinoma, melanoma, lymphoma, adenocarcinoma, metastatic tumors, urothelial carcinoma, neuroendocrine carcinoma, Merkel cell carcinoma, and sarcomas.

Penile adenocarcinomas are frequently metastases from a primary tumor of pelvic origin (genitourinary or recto-sigmoid) or, less frequently, of distant origin (pancreas or lung) [6]. Clinical manifestations typically include skin lesions, malignant priapism, and skin or penile nodules.
Here we describe a case of penile adenocarcinoma with no other primary tumor. This case presented with acute urinary retention due to extrinsic urethral obstruction.

\section{Case report}

A 53-year-old man presented to the emergency department due to an episode of acute urinary retention. The patient had experienced a weight loss of $17 \mathrm{~kg}$ over the last 6 months without loss of appetite and was generally in a good condition. The patient's medical history included heavy smoking, and an in-situ Clark 1 melanoma on his back that had been completely resected. The patient did not present any external lesion (Figure 1A and 1B)

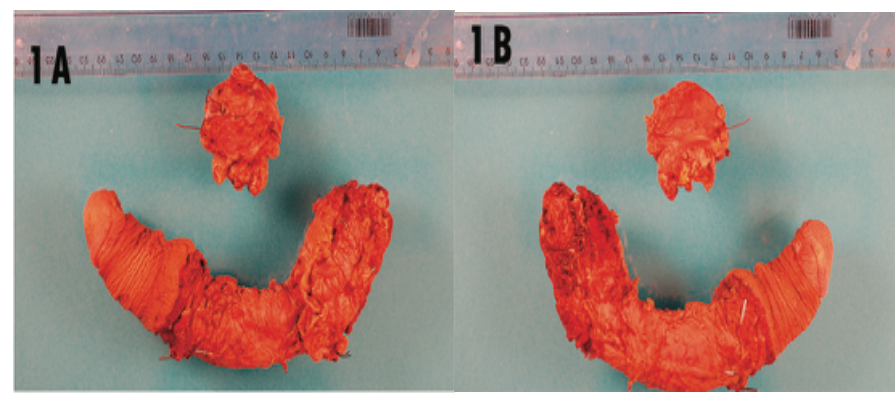

Figure 1. Macroscopic Aspect of the Surgical Specimen with No Macroscopic Externa Lesion

${ }^{\star}$ Correspondence to: Marcelo Di Gregorio, Department of Urology, CHU UCL Namur site-Godinne, Belgium, Tel: 003281423478; E-mail: marcelo.digregorio@ uclouvain.be

Key words: Penis tumor, Primary adenocarcinoma, adult urinary retention, case report

Received: March 29, 2020; Accepted: April 06, 2020; Published: April 09, 2020 
Penile ultrasound revealed heterogeneous nodules in the cavernous body and in the spongy body (Figure 2A). Biopsy of the nodules within the cavernous body of the penis revealed adenocarcinoma (Figure 2B). Carcinoma of a transient character was excluded by the immunohistochemical profile, and negative expression of PSA, PSAP, and CEA.

Whole-body fluorodeoxyglucose-positron emission tomography (FDG-PET) revealed multiple hypermetabolic focal penile nodules associated with hypermetabolic lymph nodes of the inguinal region, without any abdominal or thoracic pelvic lesions (Figure 2C). Laboratory findings and tumoral markers, including prostate-specific antigen (PSA), CEA carcinoembryonic antigen (CEA), beta-human chorionic gonadotropin (bHCG), and alpha-fetoprotein, were normal.

The patient was started on neo-adjuvant chemotherapeutic treatment with carboplatin and paclitaxel, administered weekly, for four courses over 12 weeks. Three months later, MRI showed the disappearance of inguinal nodes, but persistence of suspicious focal spots in the penis (Figure 2D and 2E).

A multidisciplinary staff team decided to complete the treatment with surgery. The patient underwent radical penectomy, prostatectomy, and enlarged lymphadenectomy associated with a Mitrofanoff-type urinary diversion. Microscopic examination revealed non-residual tumoral tissue, and fibrotic scars dissecting the parenchyma around the corpora cavernosa and corpus spongiosum (Figure $2 \mathrm{~F}$ ).

Twenty months later, the patient presented with left amaurosis. Cerebral MRI showed a 4-cm frontal lesion and a smaller (about 2 $\mathrm{cm}$ ) right fronto-parietal lesion (Figure 2B). Exeresis of the frontal lesion revealed an adenocarcinoma metastasis with poor-to-moderate differentiation (Figure $3 \mathrm{~A}$ and $3 \mathrm{~B}$ ). The patient underwent stereotactic radiation therapy to the right fronto-parietal lesion, with $23.3 \mathrm{~Gy}$ administered in three 7.7-Gy fractions.

Three months later, a follow-up brain MRI revealed three left frontal extra-axial lesions (pachymeningeal dissemination). Radiotherapy of the pan-encephalic region and cervical medullary canal up to C4 was performed (30 Gy, $10 \times 3 \mathrm{~Gy}$ ), and immunotherapy (nivolumab) was

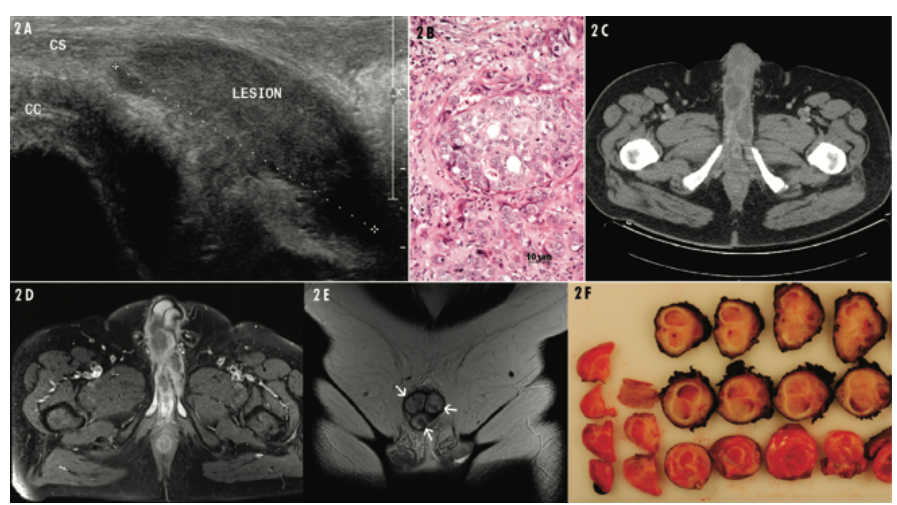

Figure 2. Imaging and Histological Analyses of Penile Adenocarcinoma

Panel A shows the ultrasound ventral longitudinal view of the penis, revealing a large hypoechoic lesion centered in the spongious body (CS). The base of the cavernous body is partly visible (CC). Panel B shows the morphological aspects of the initial lesion, which correspond to adenocarcinoma (H\&E staining, magnification $\times 20$ ). Panel $\mathrm{C}$ shows the axial contrast-enhanced multidetector computed tomography image at the level of the penis, revealing multiple hypodense nodules with peripheral enhancement of both cavernous bodies. Panel D shows the axial T1-weighted MRI of the penis after gadolinium injection, revealing multiple nodules with peripheral enhancement of both cavernous bodies. Panel E shows the T2-weighted transverse view of the penis, revealing the lesions of both cavernous bodies and in the spongious body (white arrows). Panel F shows the macroscopic penile sections. Non-residual tumoral tissue can be observed. Fibrotic scars dissect the parenchyme around the corpora cavernosa and corpus spongiosum.
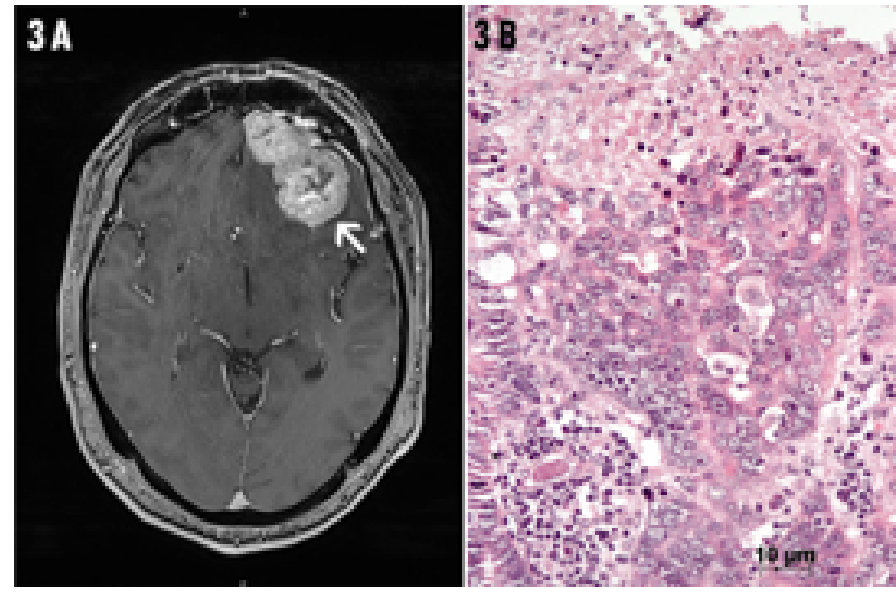

Figure 3. Imaging and Histological Analyses of Cerebral Metastasis

Panel A shows the axial T1-weighted brain MRI after gadolinium injection, revealing a left frontal enhancing lesion (arrow). Panel B shows the morphological aspects of the cerebral metastasis, which correspond to adenocarcinoma with a histological presentation very similar to the primary penile lesion (H\&E staining, magnification $\times 20$ ).

initiated. Unfortunately, the patient progressed and ultimately died at home. No autopsy was performed.

\section{Discussion}

Penile tissue contains several types of cells, and different types of penile cancer can develop from each cell type. Erreur ! Signet non défini $1^{1}$. Non-squamous-cell carcinomas of the penis are rare lesions, which can include basal cell carcinoma [7], adenocarcinoma [8], and adenosquamous carcinoma [9]. Most penile adenocarcinomas arise in the periurethral glands of Littre [10], Cowper glands, and sweat glands of the shaft [11-12]. Such tumors typically originate from other organs and are considered a metastatic disease.

To our knowledge, primary adenocarcinoma of the penis has been previously described in a dog [13] and only in one human report by Van Savage, et al. in 1994; however, that case had a different clinical presentation (fungating lesion of the penis glans) [6]. In the presently reported case, the patient didn't present any external lesion. The initial symptom was acute urinary retention due to extrinsic urethral obstruction. It is unlikely that this tumor originated in a preputial gland on the inner layer of the prepuce (glands of Tyson). Primary adenocarcinoma of the penis may arise from penile glands, such as eccrine sweat glands or meatal urethral glands [14].

Most previously reported adenocarcinomas of the penis have been identified as metastatic lesions, the majority being found in the corpora cavernosa [15,16] and exceptional in the corpus spongiosum [17]. Adenocarcinoma metastasis to the penis is most often reported from primary tumors in the bladder (30.6\%), prostate (29\%), and rectumsigmoid (14\%) [16]. However, other primary sites have also been described, including the kidney, hematologic system, lung, and testis [16]. Such metastases most commonly occur in cases with a previously identified and substantial primary mass. The patient in our present case underwent initial physical, biochemical, radiological examinations to exclude any such primary source, and remained free of any other apparent tumors for 15 months. No primary adenocarcinoma outside of the penis was ever found in our patient. The patient was regularly monitored for recurrent disease and ultimately progressed, showing cerebral metastases.

${ }^{1} \mathrm{Al}$ Arsh Basheer (2018) Chemical chiral pollution: Impact on the society and science and need of the regulations in the 21 st century $30: 319-522$. 
The diagnosis of a primary penile tumor is difficult, requiring exclusion of the possibility that the penile lesion is secondary to any prior lesion of multiple possible origins. A secondary metastatic lesion is the most frequent cause of penile adenocarcinoma, which in itself is a rare pathology. In our present case, all the tumor markers that might have indicated a possible primary tumor of digestive or urinary origin were negative. Despite the patient's history of prior melanoma, immunostaining for a possible origin of melanoma was also negative. Multiple imaging tests (ultrasound, FDG-PET, and MRI) were also negative in the search for a non-penile primary tumor. Bladder cystoscopy and urinary cytology at the time of cerebral metastasis diagnosis were also negative.

Penile secondary malignancy is usually associated with disseminated disease in which every metastatic mechanism could play a key role. Each different primary tumor tends to metastasize according to different patterns, including direct extension (continuity, contiguity), lymphatic system, arterial spread venous route, or iatrogenic implantation [6]. Our patient did not present lesions of dermatitis or erythema, which would be expected in extramammary Paget's disease (EMPD) $[12,18]$ Moreover, we did not find Toker cells in the examined lesion samples.

Treatment options for penile adenocarcinoma include total penectomy with locoregional lymphadenectomy, and consolidation chemotherapy to avoid local or distant recurrence. Adenocarcinomas respond poorly to the latter options, decreasing the chances of disease control and complicating the management of recurrence disease. Cisplatin-based chemotherapy has been useful for some adenocarcinomas of other origins, and it has also been used to treat metastatic diseases. New Platinum compound (carboplatin, oxaliplatin, nedaplatin, lobaplatin, heptaplatin) are new perspectives in the cancer treatment [19]. In our case, treatment included radical penectomy with prostatectomy, with ilioobturator lymphadenectomy and consolidation carboplatin chemotherapy.

Median survival of patients with metastatic adenocarcinoma of the penis is 4 to 24 months for prostatic primary disease, 5 to 22 months for primary colorectal malignancy, and 2 to 12 months for primary renal malignancy. The patient in our present case died 25 months after the initial diagnosis, despite multiple different treatments. The development of nano-anticancer drugs should be life expectancy since the matter in nano regime show excellent properties.

Nano regimes of the drugs increase their bioavailability, side specific delivery and exhibits non or little side effects for normal cells with potential to cross the barriers of cancer cells easily $[3,20]$.

Let's hope and wish for the revolution of chemotherapy in providing relief to the people suffering from rare deadly fatal cancer $[19,20]$.

\section{Conclusion}

Here we describe an unusual case of acute urinary retention due to a very rare primary tumor of the penis. Despite an early diagnosis, aggressive treatment, and frequent and exhaustive follow-up, the disease aggressiveness led to short survival.

\section{References}

1. Al Arsh Basheer (2018) Chemical chiral pollution: Impact on the society and science and need of the regulations in the 21 st century $30: 319-522$

2. Jemal A, Bray F, Center MM, Ferlay J, Ward E, et al. (2011) Global cancer statistics CA Cancer J Clin 61: 69-90. [Crossref]

3. Ali I, Nadeem Lone M, Suhail M, Danish Mukhtar S, Asnin L (2016) Advances in nanocarriers for anticancer drugs delivery. Current medicinal chemistry 23: 2159-2187.

4. Ali I, Nadeem Lone M, A Al-Othman Z, Al-Warthan A, Marsin Sanagi M (2015) Heterocyclic scaffolds: centrality in anticancer drug development. Current drug targets 16: 711-734.

5. Sharma P, Zargar-Shoshtari K, Pettaway CA, Schabath MB, Giuliano AR, et al. (2016) Disparities in penile cancer. Cancer Control 23: 409-414.

6. Mearini L, Colella R, Zucchi A, Nunzi E, Porrozzi C, et al. (2012) A review of penile metastasis. Oncology reviews 6: 1.

7. Kim ED, Kroft S, Dalton DP (1994) Basal cell carcinoma of the penis: case report and review of the literature. J Urol 152: 1557-1559. [Crossref]

8. Van Savage JG, Carson CC (1994) Primary adenocarcinoma of the penis. J Urol 152 1555-1556. [Crossref]

9. Cubilla AL, Ayala MT, Barreto JE, Bellasai JG, Nöel JC (1996) Surface adenosquamous carcinoma of the penis: a report of three cases. The American journal of surgical pathology 20: 156-160.

10. Dubos EI, SW Downing, SM Prather Ashe (1954) Primary carcinoma originating in the Littre glands. Cancer 7: 539-548

11. Carpenter AA, Bernardo JR Jr (1971) Adenoid cystic carcinoma of Cowper's gland: case report. J Urol 106: 701-703. [Crossref]

12. Mitsudo S, Nakanishi I, Koss LG (1981) Paget's disease of the penis and adjacent skin its association with fatal sweat gland carcinoma. Archives of pathology \& laboratory medicine 105: 518.

13. Furtado ARR, Parrinello L, Merlo M, Di Bella A (2015) Primary penile adenocarcinoma with concurrent hypercalcaemia of malignancy in a dog. Journal of Small Animal Practice 56: 289-292.

14. Layfield LJ, Liu K (2000) Mucoepidermoid carcinoma arising in the glans penis. Archives of pathology \& laboratory medicine 124: 148-151.

15. Dubocq FM, Tefilli MV, Grignon DJ, Pontes JE, Dhabuwala CB (1998) High flow malignant priapism with isolated metastasis to the corpora cavernosa. Urology 51: 324 326.

16. De Luca F, Zacharakis E, Shabbir M, Maurizi A, Manzi E, et al. (2016) Malignan priapism due to penile metastases: Case series and literature review. Archivio Italiano di Urologia e Andrologia 88: 150-152.

17. Gorospe L, González-García A, González-Gordaliza MC, Gómez-Dos-Santos V, García-Gómez-Muriel I, et al. (2018) Metastasis to the corpus spongiosum of the penis in a patient with lung cancer causing acute urine retention. Surgery 163: 474-475.

18. Hashemi P, Kao GF, Konia T, Kauffman LC, Tam CC, et al. (2014) Multicentric primary extramammary Paget disease: a Toker cell disorder? Cutis 94: 35-38.

19. Ali I, A Wani W, Saleem K, Haque A (2013) Platinum compounds: a hope for future cancer chemotherapy. Anti-Cancer Agents in Medicinal Chemistry 13: 296-306.

20. Ali I, Mukhtar SD, Hsieh MF, Alothman ZA, Alwarthan A (2018) Facile synthesis of indole heterocyclic compounds based micellar nano anti-cancer drugs. RSC advances 8: $37905-37914$

Copyright: (C2020 Marcelo Di Gregorio. This is an open-access article distributed under the terms of the Creative Commons Attribution License, which permits unrestricted use, distribution, and reproduction in any medium, provided the original author and source are credited. 